\title{
Evaluación económica del tratamiento médico de la hiperplasia benigna de próstata (HBP) en España en atención especializada. Aplicación al coste- efectividad de dos fármacos habitualmente utilizados en su tratamiento
}

\author{
Carballido $\mathrm{J}^{1}$, Ruiz-Cerdá $\mathrm{JL}^{2}$, Unda $\mathrm{M}^{3}$, Baena $\mathrm{V}^{4}$, Campoy $\mathrm{P}^{5}$, Manasanch $\mathrm{J}^{6}$, Slof $\mathrm{J}^{7}$. \\ ${ }^{1}$ Servicio de Urología Hosp. Univ. Puerta de Hierro, Madrid. ${ }^{2}$ Servicio de Urología Hosp. Univ. La Fe, Valencia. \\ ${ }^{3}$ Servicio de Urología Hosp. de Basurto, Bilbao. ${ }^{4}$ Servicio de Urología Hosp. Univ. Carlos Haya, Málaga. \\ ${ }^{5}$ Servicio de Urología Hosp. Univ. Virgen del Rocio, Sevilla. ${ }^{6}$ Pierre Fabre Ibérica S.A., Barcelona. \\ ${ }^{7}$ Departamento de Economía de la Empresa. Univ. Autónoma de Barcelona.
}

Actas Urol Esp. 2008;32(9):916-925

\section{RESUMEN}

EVALUACIÓN ECONÓMICA DEL TRATAMIENTO MÉDICO DE LA HIPERPLASIA BENIGNA DE PRÓSTATA (HBP) EN ESPAÑA EN ATENCIÓN ESPECIALIZADA. APLICACIÓN AL COSTE-EFECTIVIDAD DE DOS FÁRMACOS HABITUALMENTE UTILIZADOS EN SU TRATAMIENTO

Objetivos: Llevar a cabo un estudio farmacoeconómico para conocer el coste medio del diagnóstico y seguimiento de la HBP en España en el ámbito de la atención especializada desde la perspectiva del sistema público de salud, considerando dos fármacos frecuentemente utilizados en el entorno sanitario español, un alfabloqueante (tamsulosina) y el extracto lipidoesterólico de Serenoa repens (Permixón ${ }^{\circledR}$ ).

Material y métodos: Se determinaron los costes sanitarios directos del diagnóstico y tratamiento de la HBP para cada presentación clínica según el valor del International Prostate Symptom Score (IPSS): leve, moderada y grave. Los datos sobre el consumo y los costes unitarios de los recursos sanitarios se recogieron mediante una encuesta semi-estructurada a expertos clínicos. La eficacia clínica del tratamiento médico fue obtenida del estudio clínico PERMAL, en el que se observó equivalencia terapéutica entre ambos fármacos.

Resultados: El coste medio anual de pruebas diagnósticas y visitas médicas de la HBP sintomática según fuese leve, moderada o grave fueron, respectivamente, $124 €, 207 €$ y $286 €$ para pacientes tratados en atención especializada.

El coste medio anual del tratamiento médico, incluyendo la atención de los efectos adversos fue de $211 €$ para Permixón ${ }^{\circledR}$ y $346 €$ para tamsulosina.

Discusión: El coste de la atención médica de la HBP es proporcional a la intensidad de la sintomatología. El tratamiento farmacológico constituye una parte significativa del coste de la enfermedad. En base al modelo utilizado, el tratamiento con Permixón ${ }^{\circledR}$ es sensiblemente más coste-efectivo que el tratamiento con tamsulosina, representando un ahorro medio de $135 €$ por paciente y año.

Palabras clave: Análisis coste-efectividad. Hiperplasia benigna de próstata. Tratamiento médico. Gestión de recursos. Serenoa repens. Tamsulosina.

\section{ABSTRACT}

ECONOMIC EVALUATION OF MEDICAL TREATMENT OF BENIGN PROSTATIC HYPERPLASIA (BPH) IN THE SPECIALISED CARE SETTING IN SPAIN. APPLICATION TO THE COST-EFFECTIVENESS OF TWO DRUGS FREQUENTLY USED IN ITS TREATMENT

Objectives: To develop a pharmacoeconomic study in order to know the average cost of BPH diagnosis and follow-up in Spain in the Urology Department setting from the perspective of the public health system, considering two frequently used drugs in the Spanish Healthcare environment, an alpha-blocker (tamsulosin) and the lipido-sterolic extract of Serenoa repens (Permixon ${ }^{\circledR}$ ).

Material and methods: Direct healthcare costs of BPH diagnosis and treatment were determined for each clinical stage according to the International Prostate Symptom Score (IPSS): mild, moderate and severe. Data on the usage and unit costs of healthcare resources were obtained from a semi-structured interview with clinical experts. The clinical efficacy of the medical treatments was obtained from the PERMAL clinical study, where therapeutic equivalence between the two studied drugs was observed.

Results: For patients treated in the Urology Department setting, the average annual cost of diagnostic tests and medical visits related to mild, moderate or severe BPH symptoms were, respectively, € 124, € 207, and € 286 .

The average annual cost of the drugs, including adverse effects treatment, was $€ 211$ for Permixon ${ }^{\circledR}$ and $€ 346$ for tamsulosin.

Discussion: Costs of medical care of BPH increases with symptom intensity. Pharmacological treatment makes up a significant part of the disease's cost. According to the model used, treatment with Permixon ${ }^{\circledR}$ is considerably more cost-effective than with tamsulosin, offering average yearly savings of $€ 135$ per patient.

Keywords: Benign prostatic hyperplasia. Cost effectiveness analysis. Drug therapy. Healthcare resources. Serenoa repens. Tamsulosin.

Este estudio ha sido realizado gracias a una beca de Pierre Fabre Ibérica S.A. 
$\mathrm{L}^{\mathrm{a}}$ a hiperplasia benigna de próstata (HBP) es una Aenfermedad relacionada con la dependencia androgénica y con la edad, caracterizada por la proliferación del tejido prostático que puede progresar hacia una retención urinaria aguda y que a menudo se asocia con una disminución de la calidad de vida $^{1}$. Su prevalencia aumenta con la edad, siendo especialmente frecuente después de los 50 años ${ }^{2,3}$. Efectivamente, la HBP es la primera causa de consulta en los servicios de urología y la segunda causa de ingreso para intervención quirúrgica ${ }^{4}$. Considerando que en España hay actualmente más de 6.650.000 españoles mayores de 50 años, se estima que más de 1.500 .000 hombres precisarian de atención médica anual debido a procesos prostáticos ${ }^{5}$.

Existe una amplia gama de opciones terapéuticas ante la HBP, que van desde la vigilancia expectante y los tratamientos farmacológicos a los tratamientos quirúrgicos con diferente nivel de invasivi$\mathrm{dad}^{4,6}$. La carga económica de estos tratamientos es sustancial en términos de costes sanitarios directos; por ejemplo, recientemente se estimó que el coste anual de los tratamientos de HBP en España había pasado de 17,5 millones de euros en el año 1992 a 55 millones en el año $1998^{4}$. A esto se añaden costes indirectos asociados, como pérdidas de productividad laboral e interrupciones de las actividades cotidianas.

En el entorno sanitario español los costes relativos al diagnóstico y tratamiento de las enfermedades están adquiriendo una importancia creciente. Esta circunstancia es especialmente relevante en aquellas situaciones en las que la distribución de recursos es prioritaria y en enfermedades de elevada incidencia y prevalencia como la HBP. No obstante, el coste de la HBP y la relación costeefectividad de las diferentes opciones terapéuticas para tratarla siguen siendo mal conocidos, particularmente en el contexto del sistema sanitario español, lo que dificulta optimizar las estrategias de actuación ${ }^{7}$.

En evaluación económica de la salud se diferencian frecuentemente tres niveles de costes relacionados con los tratamientos médicos: los costes directos, los costes indirectos y los costes intangibles. Los costes directos son los relacionados con el uso de recursos sanitarios directamente relaciona- dos con el tratamiento de una enfermedad (fármacos, horas de personal sanitario, pruebas diagnósticas, materiales, visitas, etc.), los costes indirectos se refieren a pérdidas de productividad del paciente o sus cuidadores (por incapacidad temporal o permanente o muerte) y los costes intangibles se refieren a aspectos a los que es difícil asignar una valor monetario, tales como el dolor, la pérdida de calidad de vida o el sufrimiento relacionados con la enfermedad o su tratamiento ${ }^{8}$.

El objetivo del presente estudio es aportar datos que sean de utilidad en este sentido. Así, se pretende determinar, en primer lugar, el coste medio del diagnóstico y seguimiento de la HBP en España, atendiendo al grado de intensidad de la sintomatología y el nivel asistencial donde se prestan los servicios sanitarios, y en segundo lugar, el coste-efectividad de su tratamiento médico con dos fármacos ampliamente utilizados en el entorno sanitario español, un alfabloqueante (tamsulosina) y el extracto lipidoesterólico de Serenoa repens (Permixón ${ }^{\circledR}$ ), basado en los resultados de un estudio clínico aleatorizado, comparativo, de un año de duración ${ }^{9}$, que incorpora los datos necesarios para evaluar la eficacia clínica y la tolerabilidad de los medicamentos estudiados.

\section{MATERIAL Y MÉTODOS}

Planteamiento general y perspectiva del estudio

Se estima el coste del diagnóstico y seguimiento de pacientes con HBP en atención especializada, según presenten sintomatología leve, moderada o grave, así como el coste anual y el coste-efectividad del tratamiento médico de la HBP con dos fármacos de uso frecuente en el sistema sanitario español. Los datos necesarios para esta estimación se obtuvieron principalmente a través de un cuestionario dirigido a un panel de médicos especialistas en urología con amplia experiencia en el tratamiento de la HBP.

El estudio se realiza desde la perspectiva del sistema público de salud (SPS) en España. El análisis se ciñe a los costes sanitarios directos soportados por el SPS en centros y hospitales que prestan tratamiento a los pacientes con HBP y de la prestación farmacéutica pública relacionada.

El diagnóstico y seguimiento de la HBP leve y moderada también se lleva a cabo por parte de los 
médicos de atención primaria en un número importante de casos. De hecho, en alguna comunidad autónoma (p. ej. Andalucía) la HBP leve, en el ámbito de la sanidad pública, es responsabilidad exclusiva del médico de atención primaria. Por este motivo, y con la finalidad de ofrecer una visión global y complementaria respecto al diagnóstico y tratamiento médico de la $\mathrm{HBP}$, se han recabado datos relativos a la actividad asistencial de médicos de atención primaria en la HBP leve y moderada.

El estudio clínico en el que se basa este análisis ${ }^{9}$ informa de una buena tolerabilidad para ambos fármacos estudiados, el extracto lipidoesterólico de Serenoa repens y la tamsulosina, salvo en una significativamente superior incidencia de trastornos de la eyaculación en el grupo tratado con tamsulosina $(4,2 \%)$. Debido a que dicha diferencia no parece que debiese afectar, de forma muy importante, a la calidad de vida, no se ha planteado la realización de un estudio coste-utilidad que, en su caso, incorporaría el análisis de la seguridad global de los medicamentos estudiados.

\section{Clasificación de pacientes}

Este estudio presenta resultados estratificados por la gravedad de la sintomatología de la HBP. A este fin, se clasificaron los pacientes en base al International Prostate Symptom Score (IPSS). Este instrumento, que ha sido validado en diferentes paí$\operatorname{ses}^{10,11}$ y en diversas situaciones sociodemográficas y estados de salud ${ }^{12}$, asigna una puntuación entre 0 y 35 puntos, y cataloga los pacientes como portadores de sintomatología leve (0-7 puntos), moderada (8-19 puntos) o grave (20-35 puntos).

\section{Consumo y coste unitario de los recursos sanitarios}

El coste del diagnóstico y seguimiento de un paciente se obtiene multiplicando el coste unitario de cada recurso sanitario utilizado por el número de unidades consumidas. En este estudio se obtuvieron los datos sobre el consumo de los recursos sanitarios mediante una encuesta semi-estructurada a expertos clínicos de un total de nueve centros españoles, y que fue administrada durante el año 2005 (Anexo 1). En concreto, se consultaron a siete especialistas urólogos y a dos centros de atención primaria que complementaron la información ofrecida por los primeros respecto a los grados leve y moderado de la HBP. Los participantes fueron seleccionados con la finalidad de reflejar la práctica de diferentes áreas geográficas del territorio español y distintos niveles de atención médica.

Se solicitaron datos sobre la realización de pruebas diagnósticas y visitas médicas en los pacientes afectos de HBP que presentaran sintomatología leve, moderada o grave. No se incluyeron datos relacionados con el tratamiento quirúrgico al no ser este uno de los objetivos del estudio. Para cada tipo de recurso se pidió el porcentaje de pacientes que requiere el uso del mismo y, en su caso, el número de veces al año que se utiliza. Para cada nivel de gravedad y cada nivel asistencial, se determinó posteriormente la media, la mediana, el máximo y el mínimo de las respuestas obtenidas. A todos los entrevistados, se les solicitó que facilitaran datos referentes a la práctica habitual llevada a cabo en su centro de trabajo en aquellos pacientes con sospecha de estar afectados de HBP en cuanto a su diagnostico inicial y posterior seguimiento.

También los costes unitarios de las pruebas diagnósticas y de las visitas médicas se obtuvieron de los datos aportados por los especialistas en urología participantes en el estudio.

\section{Fármacos comparados}

Las guías clínicas recomiendan iniciar el tratamiento farmacológico en pacientes con sintomatología moderada y en aquellos que presentan sintomas más graves pero que, en ausencia de complicaciones, no deseen someterse a un tratamiento quirúrgico, así como en aquellos pacientes cuya sintomatología, aún siendo leve, afecte marcadamente a su calidad de vida ${ }^{4,6,13}$.

En la actualidad hay tres grupos farmacológicos autorizados por la Agencia Española del Medicamento y Productos Sanitarios para el tratamiento de los síntomas del tracto urinario inferior relacionados con la HBP: alfabloqueantes, inhibidores de la enzima 5-alfarreductasa y algunos fármacos fitoterapéuticos. En este estudio se analizan dos de estos fármacos, el alfabloqueante tamsulosina y un extracto lipidoesterólico de Serenoa repens (Permixón ${ }^{\circledR}$ ). Ambos medicamentos son frecuentemente utilizados en el entorno sanitario español. 


\section{Eficacia clínica de los tratamientos} farmacológicos comparados

Para valorar la relación coste-efectividad de diferentes alternativas de tratamiento es necesario conocer su coste pero también su efectividad clínica. La eficacia clínica del tratamiento y la incidencia de efectos adversos de los dos fármacos comparados en este trabajo fueron obtenidas del estudio PERMAL $^{9}$.

En este estudio clínico realizado a doble ciego, aleatorizado y multicéntrico, comparativo entre tamsulosina, 0,4 mg/día p.o., y Permixón, 360 mg/día p.o., de un año de duración, participaron 704 pacientes con una edad comprendida entre 50 y 85 años (edad media: 65 años), que presentaban un IPSS $\geq 10$ puntos, un flujo urinario máximo (Qmax) entre 5 y $15 \mathrm{ml} / \mathrm{s}$, un volumen prostático $\geq 25 \mathrm{cc}$, un volumen residual menor de $150 \mathrm{ml}$ y un PSA $<4$ ng/ml y si entre 4 y $10 \mathrm{ng} / \mathrm{ml}$, debían presentar una relación PSA libre/total $\geq 15 \%$.

En el estudio PERMAL se observó equivalencia terapéutica (en términos de IPSS y Qmax) entre ambos medicamentos, con una menor incidencia de acontecimientos adversos para Permixón, estadísticamente significativa, en el apartado de alteraciones de la eyaculación.

\section{Coste de los fármacos comparados}

El coste de los fármacos comparados en este estudio se basó en el precio de venta al público (PVP) con el 4\% de IVA de acuerdo con el Catálogo de Especialidades Farmacéuticas ${ }^{14}$. Dado que estos fármacos están disponibles en un número considerable de presentaciones diferentes, se ponderaron los precios según el volumen de ventas de cada presentación en el año 2006, según la información obtenida por la empresa IMS Health para los fármacos analizados en este estudio y para dicho año. A partir del precio medio ponderado por mg, se calculó el coste de la dosis diaria recomendada para el tratamiento de los síntomas relacionados con la HBP (tamsulosina: 0,4 mg/día; Permixón ${ }^{\circledR}: 320 \mathrm{mg} /$ día), y se obtuvo el coste anual del tratamiento farmacológico multiplicando este dato por 365 días.

Al no disponerse de datos fidedignos que evalúen en nuestro medio el coste derivado de la atención de los acontecimientos adversos provocados por los fármacos en estudio cuando se utilizan en el tratamiento de los síntomas de la HBP, no se pudo imputar el coste exacto relacionado con el tratamiento de dichos acontecimientos adversos. No obstante, con la finalidad de no subestimar completamente su impacto se supuso que el coste sanitario mínimo asociado sería el de una visita del paciente al médico de atención primaria con el objetivo de consultar acerca de la presencia del acontecimientos adverso en cuestión. Por tanto, el coste iatrogénico se estimó para cada uno de los dos fármacos multiplicando la tasa de incidencia de efectos adversos por el coste de una visita, donde las incidencias de cada efecto se basaron en el ya mencionado estudio PERMAL 9 .

\section{Análisis de sensibilidad}

Dada la dispersión en los datos obtenidos de los distintos centros respecto al diagnóstico y seguimiento de la HBP, se llevó a cabo un análisis de sensibilidad univariante, variando la cantidad y el coste unitario de los recursos sanitarios consumidos entre los mínimos y máximos señalados por los expertos consultados. Dado que las cantidades y costes unitarios de los recursos sanitarios consumidos no parecían distribuirse de forma simétrica, también se volvieron a calcular los resultados utilizando la mediana de las respuestas obtenidas, en vez de su media aritmética. Respecto a los costes farmacológicos, la principal fuente de incertidumbre es la posible mayor sustitución, en el futuro, de tamsulosina de marca por su genérico. Por tanto, se consideró como escenario alternativo extremo la utilización de tamsulosina genérica en el 100\% de los casos.

\section{RESULTADOS \\ Consumo y coste unitario de recursos sanitarios}

Las Tablas 1 y 2 muestran el uso de recursos sanitarios según la gravedad de la sintomatología para pacientes tratados en atención especializada y en atención primaria, respectivamente. Es preciso señalar que los resultados relativos a la HBP leve en la Tabla 1 incorporan exclusivamente las respuestas de los especialistas que han indicado que son consultados por pacientes con síntomas leves, lo que no ocurre en todos los casos del panel consultado. La Tabla 2 no incorpora los costes relacionados con la HBP grave en atención primaria dado que 
Tabla 1. Consumo anual de recursos sanitarios de pacientes tratados en atención especializada*

\begin{tabular}{|c|c|c|c|c|c|c|c|c|c|c|c|c|}
\hline \multirow{3}{*}{$\begin{array}{l}\text { TIPO DE } \\
\text { RECURSO }\end{array}$} & \multicolumn{4}{|c|}{ Pacientes HBP leve } & \multicolumn{4}{|c|}{ Pacientes HBP moderada } & \multicolumn{4}{|c|}{ Pacientes HBP grave } \\
\hline & \multicolumn{2}{|c|}{$\%$ pacientes } & \multicolumn{2}{|c|}{ veces / año } & \multicolumn{2}{|c|}{$\%$ pacientes } & \multicolumn{2}{|c|}{ veces / año } & \multicolumn{2}{|c|}{$\%$ pacientes } & \multicolumn{2}{|c|}{ veces / año } \\
\hline & promedio & $($ min. - máx.) & promedio & (min. - máx.) & promedio & (min. - máx.) & promedio & (min. - máx.) & promedio & (min. - máx.) & promedio & $(\min ,-\operatorname{máx}$.) \\
\hline \multicolumn{13}{|l|}{$\begin{array}{l}\text { Pruebas } \\
\text { diagnósticas: }\end{array}$} \\
\hline Ecografia & 42 & $(0-100)$ & 1,0 & $(1-1)$ & 51 & $(0-100)$ & 1,0 & $(1-1)$ & 71 & $(0-100)$ & 1,2 & $(1-2)$ \\
\hline Análisis de orina & 62 & $(0-100)$ & 1,0 & $(1-1)$ & 64 & $(0-100)$ & 1,0 & $(1-1)$ & 83 & $(0-100)$ & 1,5 & $(1-2)$ \\
\hline Análisis de sangre & 46 & $(0-100)$ & 1,0 & $(1-1)$ & 36 & $(0-100)$ & 1,0 & $(1-1)$ & 54 & $(0-100)$ & 1,3 & $(1-2)$ \\
\hline Creatinina sérica & 66 & $(0-100)$ & 1,0 & $(1-1)$ & 50 & $(0-100)$ & 1,0 & $(1-1)$ & 69 & $(0-100)$ & 1,2 & $(1-2)$ \\
\hline PSA & 100 & $(100-100)$ & 1,0 & $(1-1)$ & 96 & $(70-100)$ & 1,0 & $(1-1)$ & 96 & $(70-100)$ & 1,1 & $(1-2)$ \\
\hline Uroflujometria & 33 & $(0-100)$ & 1,0 & $(1-1)$ & 72 & $(20-100)$ & 1,2 & $(1-2)$ & 93 & $(80-100)$ & 1,3 & $(1-2)$ \\
\hline & \multicolumn{2}{|c|}{ veces / año } & & & \multicolumn{2}{|c|}{ veces / año } & & & \multicolumn{2}{|c|}{ veces / año } & & \\
\hline & promedio & $(m i n .-m a ́ x)$. & & & promedio & (min. - máx.) & & & promedio & (min. - máx.) & & \\
\hline \multicolumn{13}{|l|}{ Visitas médicas: } \\
\hline Atención primaria & 0,4 & $(0-1)$ & & & 1,6 & $(0-6)$ & & & 2,0 & $(0-8)$ & & \\
\hline Urologia & 1,0 & $(1-1)$ & & & 1,4 & $(1-2)$ & & & 1,8 & $(1-4)$ & & \\
\hline Urgencias (RAO) & 0,0 & $(0-0)$ & & & 0,0 & $(0-0)$ & & & 0,04 & $(0,02-0,05)$ & & \\
\hline
\end{tabular}

RAO: Retención aguda de orina

* Algunos especialistas no respondieron a las preguntas del apartado HBP leve, puesto que en la práctica clínica habitual de su CCAA esta entidad es controlada por los médicos de atención primaria.

Tabla 2. Consumo anual de recursos sanitarios de pacientes tratados en atención primaria

TIPO DE RECURSO

Pacientes HBP leve

Pacientes HBP moderada

\begin{tabular}{|c|c|c|c|c|c|c|c|c|}
\hline & \multicolumn{2}{|c|}{ \% pacientes } & \multicolumn{2}{|c|}{ veces / año } & \multicolumn{2}{|c|}{ \% pacientes } & \multicolumn{2}{|c|}{ veces / año } \\
\hline & promedio & (mín. - máx.) & promedio & (mín. - máx.) & promedio & (mín. - máx.) & promedio & (min. - máx.) \\
\hline \multicolumn{9}{|l|}{$\begin{array}{l}\text { Pruebas } \\
\text { diagnósticas: }\end{array}$} \\
\hline Ecografía & 18 & $(10-25)$ & 1 & $(1-1)$ & 55 & $(50-60)$ & 1 & $(1-1)$ \\
\hline Análisis de orina & 55 & $(10-90)$ & 1 & $(1-1)$ & 63 & $(25-100)$ & 1 & $(1-1)$ \\
\hline Análisis de sangre & 70 & $(50-90)$ & 1 & $(1-1)$ & 75 & $(50-100)$ & 1 & $(1-1)$ \\
\hline Creatinina sérica & 70 & $(50-90)$ & 1 & $(1-1)$ & 100 & $(100-100)$ & 1 & $(1-1)$ \\
\hline PSA & 98 & $(95-100)$ & 1 & $(1-1)$ & 100 & $(100-100)$ & 1 & $(1-1)$ \\
\hline \multirow[t]{3}{*}{ Uroflujometría } & 0 & $(0-0)$ & - & - & 0 & $(0-0)$ & - & - \\
\hline & \multicolumn{2}{|c|}{ veces / año } & & & \multicolumn{2}{|c|}{ veces / año } & & \\
\hline & promedio & (mín. - máx.) & & & promedio & (mín. - máx.) & & \\
\hline \multicolumn{9}{|l|}{ Visitas médicas: } \\
\hline Atención primaria & 1,8 & $(1-3)$ & & & 2,5 & $(2-3)$ & & \\
\hline Urología & 0,5 & $(0-1)$ & & & 1,5 & $(1-2)$ & & \\
\hline Urgencias (RAO) & 0,0 & $(0-0)$ & & & 0,0 & $(0-0)$ & & \\
\hline
\end{tabular}

RAO: Retención aguda de orina.

este tipo de pacientes son habitualmente derivados a la atención especializada. La Tabla 3 muestra los costes unitarios de los recursos sanitarios empleados.

Se observa que el coste del manejo de la HBP aumenta con la gravedad de la sintomatología y parece ser ligeramente menor en el ámbito de la atención primaria que en la atención especializada para pacientes con sintomatología leve. Por otra parte, se pone de manifiesto una considerable variación en las prácticas clínicas, que queda reflejada en la dispersión de los valores mínimos y máximos.

\section{Coste anual de pruebas diagnósticas y visitas médicas}

En la Figura 1 se resume el coste anual medio por paciente del conjunto de pruebas diagnósticos y 
Tabla 3. Coste unitario de pruebas diagnósticas y visitas médicas en el tratamiento de la HBP

\begin{tabular}{lcc}
\hline TIPO DE RECURSO & \multicolumn{2}{c}{ COSTE (en €) } \\
\hline Pruebas diagnósticas: & promedio & (mín. - máx.) \\
Ecografía & 35,74 & $(29,01-41,45)$ \\
Análisis de orina & 3,18 & $(1,96-4,72)$ \\
Análisis de sangre & 6,98 & $(1,14-20,00)$ \\
Creatinina sérica & 0,98 & $(0,18-2,40)$ \\
PSA & 9,81 & $(4,36-16,50)$ \\
Uroflujometría & 32,25 & $(15,00-59,83)$ \\
Visitas médicas: & & \\
Atención primaria & 33,03 & $(15,00-51,06)$ \\
Atención especializada & 69,72 & $(26,97-102,14)$ \\
Urgencias (por retención & & \\
aguda de orina) & 99,03 & $(65,11-118,95)$ \\
\hline
\end{tabular}

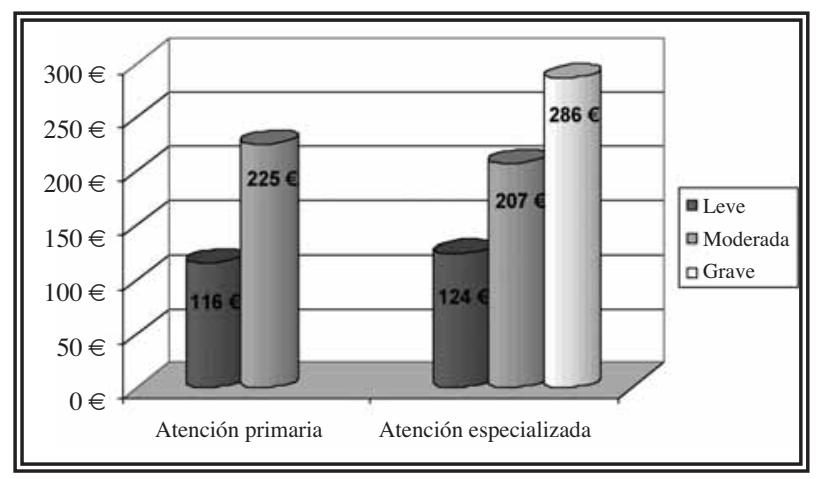

FIGURA 1. Coste anual medio de visitas médicas y pruebas analiticas de pacientes con HBP leve, moderada y grave tratados en atención especializada y en atención primaria (en € por paciente y año).

visitas médicas (sumatoria para todos los recursos de la multiplicación de las unidades consumidas por su coste unitario), según la gravedad de la sintomatología y el nivel asistencial que atiende al paciente.
La Tabla 4 muestra el análisis de sensibilidad del coste de pruebas diagnósticos y visitas médicas. En esta Tabla se puede observar cómo varían los resultados, cuando estos se calculan a partir de los valores medianos y extremos de los datos recogidos para el consumo de recursos sanitarios y sus costes unitarios. Como es esperable, la dispersión se reproduce en este análisis.

\section{Coste anual y coste-efectividad del tratamiento farmacológico con Permixón y Tamsulosina}

La Figura 2 muestra los costes anuales del tratamiento farmacológico con Permixón y tamsulosina, incluidos los costes iatrogénicos que estos generan. Comparando estos valores con los de las pruebas diagnósticas y visitas médicas, se puede constatar que el tratamiento farmacológico constituye una parte significativa del coste de la enfermedad (entre un $42 \%$ y $74 \%$, según el fármaco y tipo de paciente). El efecto de los costes iatrogénicos, por otro lado, aparece como residual en ambos casos.

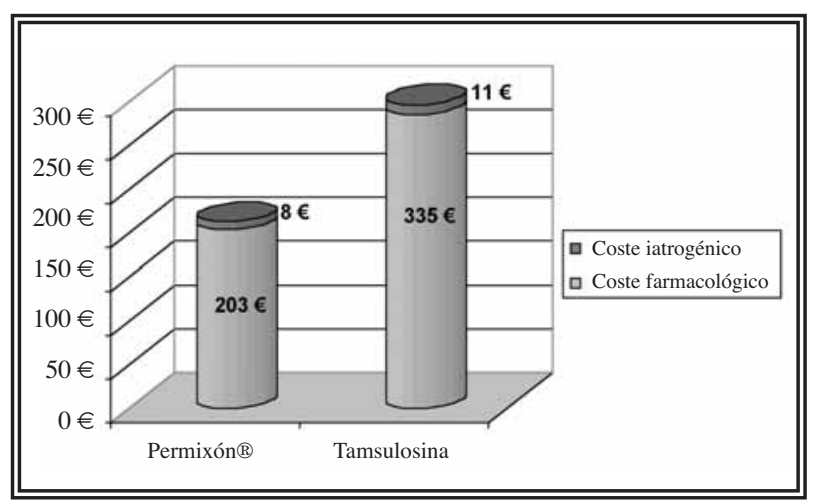

FIGURA 2. Coste del tratamiento farmacológico con Permixón ${ }^{\circledR}$ y tamsulosina (en $€$ por paciente en tratamiento farmacológico y año)

Tabla 4. Análisis de sensibilidad del coste anual medio de visitas médicas y pruebas analíticas de pacientes con HBP leve, moderada y grave tratados en atención primaria y especializada (en € por paciente y año)

\begin{tabular}{lcccccc}
\hline $\begin{array}{l}\text { Intensidad de } \\
\text { los sintomas } \\
\text { y nivel asistencial }\end{array}$ & $\begin{array}{c}\text { Escenario } \\
\text { base }\end{array}$ & $\begin{array}{c}\text { Valores } \\
\text { medianos }\end{array}$ & $\begin{array}{l}\text { Mínimo } \\
\text { consumo } \\
\text { recursos }\end{array}$ & $\begin{array}{c}\text { Máximo } \\
\text { consumo } \\
\text { recursos }\end{array}$ & $\begin{array}{c}\text { Precios } \\
\text { unitarios } \\
\text { más bajos }\end{array}$ & $\begin{array}{c}\text { Precios } \\
\text { unitarios } \\
\text { más bajos }\end{array}$ \\
\hline
\end{tabular}

\section{Atención primaria}

HBP leve

116

HBP moderada
225

124

207

286

110

110

83

132

213
50

169

80

83

104
197

51

183

281

101

192

56

726

133 
Respecto a la relación coste-efectividad, se puede observar que el coste anual del tratamiento con Permixón es $135 €$ inferior al de tamsulosina. Esto significa que Permixón representa la opción más coste-eficaz de los dos comparadores, ya que es a la vez más económico y, en base a la evidencia del estudio PERMAL, equivalente en eficacia y más tolerable que la tamsulosina (debido a la menor incidencia de acontecimientos adversos).

Si se considerara el precio de la tamsulosina genérica, Permixón seguiría siendo el fármaco más coste-eficaz en la comparación. Con el uso de Permixón se produciría un ahorro de aproximadamente $32 €$ por paciente tratado y año, lo que sigue siendo de interés para el SPS al considerar las cifras de prevalencia de la HBP en España y el impacto económico que ello implica a nivel nacional.

\section{DISCUSIÓN}

La HBP presenta una incidencia y prevalencia elevadas que se estima que aumenten al producirse un mayor envejecimiento de la población española. Debido a la carga económica que ello puede suponer para el SPS, el hecho de disponer de evaluaciones económicas que faciliten la toma de decisiones acerca de cuál es el tratamiento más adecuado en cada caso puede ser de gran ayuda.

Este es el primer estudio del que tenemos conocimiento que aporta información sobre la carga económica del diagnóstico, tratamiento médico y seguimiento de la HBP en el contexto del sistema sanitario español, diferenciando el coste en función de la gravedad de los síntomas y ofreciendo datos de dos niveles asistenciales diferentes, atención especializada y atención primaria.

En general, los resultados obtenidos están en concordancia con otros trabajos que se interesaron por aspectos relativos a la HBP en nuestro medio ${ }^{7,15-18}$. En el estudio de Unda et $a^{16}$ el porcentaje de pacientes a los que se realiza ecografía diagnóstica $(76 \%)$ es similar al obtenido en este estudio, no obstante, la determinación del PSA se realizaba en el 54,2\% de los pacientes y prácticamente en la totalidad de los mismos en nuestro caso. Una probable explicación para esta diferencia es la evolución que esta prueba parece haber tenido en el tiempo, dado que el estudio de Unda et al se llevó a cabo en 1999. Efectivamente, una reciente comunicación basada en una encuesta llevada a cabo en 121 urólogos españoles ${ }^{19}$ señalaba que la determinación del PSA era utilizada por más del 95\% de los urólogos consultados, en su práctica clínica habitual, para el diagnóstico y seguimiento de la HBP.

Las diferencias de este estudio con el artículo de Meseguer et $\mathrm{al}^{15}$ radican en que el presente trabajo se basa en la experiencia clínica de facultativos españoles en su práctica clínica habitual y evalúa los costes diagnósticos y de seguimiento de la HBP en función de la intensidad de su sintomatología. El artículo de Meseguer se ceñía exclusivamente a la HBP de intensidad moderada, se basaba en las recomendaciones de un grupo de expertos americano y no contemplaba los costes de las pruebas diagnósticas ni de seguimiento. Aún así, este grupo encontraba un coste aproximado de $300 €$ por paciente y año para el tratamiento farmacológico (año 1998), que representa una cantidad cercana a la obtenida en el presente estudio para la HBP de grado moderado.

En comparación con estos otros trabajos, aquí se proporciona un mayor grado de detalle (al desglosar los resultados según la gravedad de la patología y nivel asistencial), una mayor precisión en la determinación del consumo de recursos sanitarios (al registrar no solamente el porcentaje de pacientes que utiliza cada recurso sino también el número de veces que lo utilizan en un año) y/o una mayor profundización en el aspecto económico (al cuantificar los consumos en términos monetarios).

Un dato destacable, y razonable, es que el coste del tratamiento de la HBP aumenta con la intensidad de la sintomatología, presentado los casos graves un coste casi tres veces superior que los leves. Aún siendo un hecho presumible, no se había contrastado ni cuantificado anteriormente. Otra evaluación interesante concierne a las diferencias encontradas entre los dos niveles asistenciales analizados.

Es importante subrayar que el coste de la medicación supone una proporción importante del manejo de la enfermedad en aquellos pacientes 
que reciben tratamiento farmacológico. Esta observación está en concordancia con los resultados encontrados por Grasa et $\mathrm{al}^{7}$ y Van Exel et $\mathrm{al}^{17}$, que situaba para España el peso del coste farmacológico dentro del coste global del manejo de la HBP (incluyendo cirugía) en un 46\%. En cualquier caso, dado el remarcable peso del coste farmacológico, podemos concluir que es necesario conocer el coste-efectividad de las alternativas farmacológicas disponibles para gestionar correctamente la carga económica que la HBP supone para el SPS.

En farmacoeconomía se considera que una opción de tratamiento es "dominante" si es igual o más eficaz y a la vez más económica que sus comparadores, o más eficaz y a la vez igual o más económica. En cambio una opción igual o menos eficaz y a la vez más cara, o menos eficaz y a la vez igual o más cara, se llama "dominada". Aplicando esta terminología al caso de la tamsulosina y Permixón, se puede constatar que este último fármaco representa la opción dominante y tamsulosina la dominada, ya que Permixón es más económico y, en base de la evidencia del estudio PERMAL, igual o más eficaz que la tamsulosina.

Ante la presencia de una opción dominante, no hay duda sobre la preferencia que debería haber por esta alternativa, siempre y cuando los datos obtenidos no tengan un margen de error que pudiera invertir el signo de las diferencias encontradas entre los comparadores y se hayan estudiado todas las alternativas. En nuestro caso concreto, referente al primer aspecto, y teniendo en cuenta la creciente evidencia al respecto ${ }^{9,20-23}$, hay motivo para expresar confianza en la afirmación que los dos fármacos comparados tienen una eficacia equivalente, con ventajas para Permixón en cuanto a tolerabilidad.

Es preciso señalar que este estudio no refleja todas las opciones de tratamiento médico disponibles quedando otras alternativas por analizar. Ello es debido a que para el cálculo de los costes para el presente estudio se parte de un estudio clínico aleatorizado con una metodología adecuada para conocer la eficacia comparativa de los dos fármacos incluidos en este estudio, a lo largo de un amplio periodo de tiempo.
Este trabajo puede presentar algunas limitaciones inherentes al contexto en el que se ha llevado a cabo. Éstas pueden relacionarse con la metodología de la recogida de datos y la base de datos para el cálculo de los costes. Así, los datos económicos obtenidos están en parte fundamentados en los costes de las áreas y/o centros en los que colaboran los expertos consultados al no disponerse de una base de datos nacional y actualizada que incorpore los costes establecidos por las distintas administraciones sanitarias en España. Otro aspecto a considerar es el tamaño muestral en relación con el número de participantes y su selección, que obedeció a su pertenencia a distintos organismos sanitarios y diferentes áreas geográficas, con el objetivo de obtener la mayor representatividad posible a nivel nacional.

Respecto a la estimación de los costes conviene indicar que se han considerado exclusivamente los costes directos. Así este análisis no ha incluido costes no sanitarios, como el coste asociado a disminución de la productividad, bajas laborales o el coste del tiempo de los acompañantes, ni ha entrado en valorar la pérdida de calidad de vida que se asocia a la enfermedad estudiada. En general, los estudios farmacoeconómicos realizados en España y desde la perspectiva del SPS no suelen incorporar dichos costes.

Finalmente, es preciso señalar que, al igual que ocurre con la mayoría de los estudios que evalúan tratamientos, los datos fueron recogidos en un periodo determinado y, por lo tanto, reflejan una imagen de la situación de aquel instante. Tanto el consumo de recursos sanitarios como sus costes unitarios pueden presentar variaciones a lo largo del tiempo.

En conclusión, el coste del manejo de la HBP parece aumentar de forma directamente proporcional a la gravedad de la sintomatología. El coste anual del tratamiento de la HBP con Permixón ${ }^{\circledR}$ es inferior al observado con tamsulosina tanto si se relaciona con una marca registrada como si se trata de tamsulosina genérica.

\section{Agradecimientos}

Agradecemos la colaboración del Dr. Antonio Alcaraz Asensio, del Dr. Francisco José Brenes Bermúdez y del Dr. Pedro Luis Reche López por sus inestimables aportaciones a este estudio. 
Anexo 1. Cuestionario semi-estructurado

Número de pruebas anuales y porcentaje de pacientes que las realizan. Indicar la frecuencia para cada grado de HBP (leve, moderado y grave)

\begin{tabular}{|l|l|l|}
\hline Estado grave & $\begin{array}{c}\text { Número de veces } \\
\text { al año }\end{array}$ & $\begin{array}{c}\text { Porcentaje de pacientes } \\
\text { que realizan la prueba }\end{array}$ \\
\hline Ecografía & & \\
\hline Análisis de orina & & \\
\hline Análisis de sangre & & \\
\hline Creatinina sérica & & \\
\hline PSA & & \\
\hline $\begin{array}{l}\text { Visitas atención } \\
\text { primaria }\end{array}$ & & \\
\hline Visitas al urólogo & & \\
\hline $\begin{array}{l}\text { Urgencias (retención } \\
\text { aguda de orina) }\end{array}$ & & \\
\hline Otras (especificar) & & \\
\hline
\end{tabular}

Coste de las pruebas diagnósticas y de las visitas médicas. Por favor, indique el coste para el centro sanitario de las siguientes pruebas diagnósticas y visitas médicas:

\begin{tabular}{|l|l|}
\hline Prueba diagnóstica & Coste de la prueba diagnóstica (€) \\
\hline Ecografía & \\
\hline Análisis de orina & \\
\hline Análisis de sangre & \\
\hline Creatinina sérica & \\
\hline PSA & \\
\hline Uroflujometría & \\
\hline Visita & Coste de la visita (€) \\
\hline Visita atención primaria & \\
\hline Visita al urólogo & \\
\hline Visita a urgencias & \\
\hline
\end{tabular}

\section{REFERENCIAS}

1. Guess HA. Epidemiology and natural history of benign prostatic hyperplasia. Urol Clin North Am. 1995;22(2):247-261.

2. Hunter DJ, Berra-Unamuno A, Martin-Gordo A. Prevalence of urinary symptoms and other urological conditions in Spanish men 50 years old or older. J Urol. 1996;155(6):1975-6.

3. Chicharro-Molero JA, Burgos-Rodriguez R, Sanchez-Cruz JJ, del Rosal-Samaniego JM, Rodero-Carcia P, Rodriguez-Vallejo JM. Prevalence of benign prostatic hyperplasia in Spanish men 40 years old or older. J Urol. 1998;159(3):878-882.

4. Blasco Valle M, Timón García A, Lázaro Muñoz V. Abordaje de la hiperplasia benigna de próstata. Actuación Aten Primaria. 2003;13(3):133-142.
5. Jiménez Cruz F. Presentación. En: Vicente Rodríguez J, editor. HBP 2001. Hiperplasia Benigna de Próstata. Madrid, Acción Médica S.A. 2001, p V.

6. Muñoz Rodríguez AM, Toribio Onieva JR, Moreno Moreno MI, Luque Vargas R. Hipertrofia benigna de próstata. Guías Clínicas 2002;2(2):1-5. Disponible en (http://www.fisterra.com).

7. Grasa Lanau V, Cuesta Alcalá JA, Ipiens Aznar A, Ansa Erice I, Aldave Villanueva J. Aproximación al coste del tratamiento médico de la hiperplasia benigna de próstata en la Comunidad Foral de Navarra. Actas Urol Esp. 2004 Apr;28(4):255-61

8. Sacristán del Castillo JA, Badía Llach X, Rovira Foros J. Farmacoeconomía: evaluación económica de medicamentos. Madrid, Editores Médicos 1995.

9. Debruyne F, Koch G, Boyle P, Da Silva FC, Gillenwater JG, Hamdy FC, Perrin P, Teillac P, Vela-Navarrete R, Raynaud JP. Comparison of a phytotherapeutic agent (Permixon) with an alpha-blocker (tamsulosin) in the treatment of benign prostatic hyperplasia: a 1-year randomised international study. Eur Urol 2002 May;41(5):497-506.

10. Bosch R, Donovan J, Okada K. Symptoms evaluation, quality of life and sexuality. IPSS. The 2nd international consultation on benign prostatic hyperplasia proceedings. En: Cockett AT, Aso Y, Chatelain C (eds). SCI 1993:131-143.

11. Vela Navarrete R, Martín Moreno JM, Calahorra FJ, Damián Moreno J, Hernández Coronado A, Boyle P.Validación cultural y lingüística, en castellano del baremo internacional de síntomas prostáticos (I-PSS). Actas Urol Esp. 1994;18(8):841847.

12. Badia X, Rodríguez F, Carballido J, García Losa M, Unda M, Dal-Ré R, Roset M; ESECI-98 Group. Influence of sociodemographic and health status variables on the American Urological Association symptom scores in patients with lower urinary tract symptoms. Urology 2001;57(1):71-77.

13. Madersbacher S, Alivizatos G, Nordling J, Sanz CR, Emberton M, de la Rosette JJ. EAU 2004 guidelines on assessment, therapy and follow-up of men with lower urinary tract symptoms suggestive of benign prostatic obstruction (BPH guidelines). Eur Urol 2004;46(5):547-554.

14. Catálogo de Especialidades Farmacéuticas. Consejo General de Colegios Oficiales de Farmacéuticos. Madrid, 2006.

15. Meseguer Barros C.M., Fidalgo García ML, Rubio Cebrían S. Análisis coste-efectividad del tratamiento de la hiperplasia prostática benigna de grado moderado. Aten Primaria 2000;25(8):546-551.

16. Unda Urzaiz M, Rodríguez Alcántara F, Badia X, García Losa M, Carballido Rodríguez J, Dal-Ré Saavedra R; Grupo ESECI-98. Características sociosanitarias y diagnósticas del paciente prostático en España a finales del siglo XX. Actas Urol Esp 2001;25(3):200-206.

17. Van Exel NJ, Koopmanschap MA, McDonnell J, Chapple CR, Berges R, Rutten FF; TRIUMPH Pan-European Expert Panel. Medical consumption and costs during a one-year follow-up of patients with LUTS suggestive of BPH in six european countries: report of the TRIUMPH study. Eur Urol 2006; 49(1):92102 .

18. Hutchison A, Farmer R, Chapple C, Berges R, Pientka L, Teillac $\mathrm{P}$ et al. Characteristics of patients presenting with LUTS/BPH in six European Countries. Eur Urol 2006;50(3):555-556.

19. Morote Robles J, Alcaraz Asensio A, Jiménez Verdejo J. Estudio de los hábitos en España sobre métodos diagnósticos y de seguimiento de la hiperplasia benigna de próstata (HBP). En: Libro de abstracts del LXX Congreso Nacional de Urología. San Sebastián, 4-7 junio, 2005; (resumen C-34). Disponible en (www.aeu.es). 
20. Aliaev IuG, Vinarov AZ, Lokshin KL, Spivak LG. Five-year experience in treating patients with prostatic hyperplasia patients with permixone (Serenoa repens "Pierre Fabre Medicament). Urologia 2002;1:23-25.

21. Pytel YA, Vinarov A, Lopatkin N, Sivkov A, Gorilovsky L, Raynaud JP. Long-term clinical and biological effects of the lipidosterolic extract of Serenoa repens in patients with symptomatic benign prostatic hyperplasia. Adv Ther 2002;19(6): 297-306.

22. Debruyne F, Boyle P, Calais Da Silva F, Gillenwater JG, Hamdy FC, Perrin P,Teillac P, et al. Evaluation of the clinical benefit of permixon and tamsulosin in severe BPH patients-PERMAL study subset analysis. Eur Urol 2004;45(6):773-779.
23. Hizli F, Uygur MC. A prospective study of the efficacy of Serenoa repens, tamsulosin, and Serenoa repens plus tamsulosin treatment for patients with benign prostate hyperplasia. Int Urol Nephrol 2007;39(3):879-886.

Correspondencia autor: Dr. John Slof Departamento de Economía de la Empresa. Universidad Autónoma de Barcelona. Campus de la UAB 08193 Bellaterra (Barcelona). Tel.: 935814 399; Fax.: 935812555 E-mail: EricJohn.Slof@uab.es Información artículo: Original - HBP Trabajo recibido: junio 2008 Trabajo aceptado: julio 2008 